
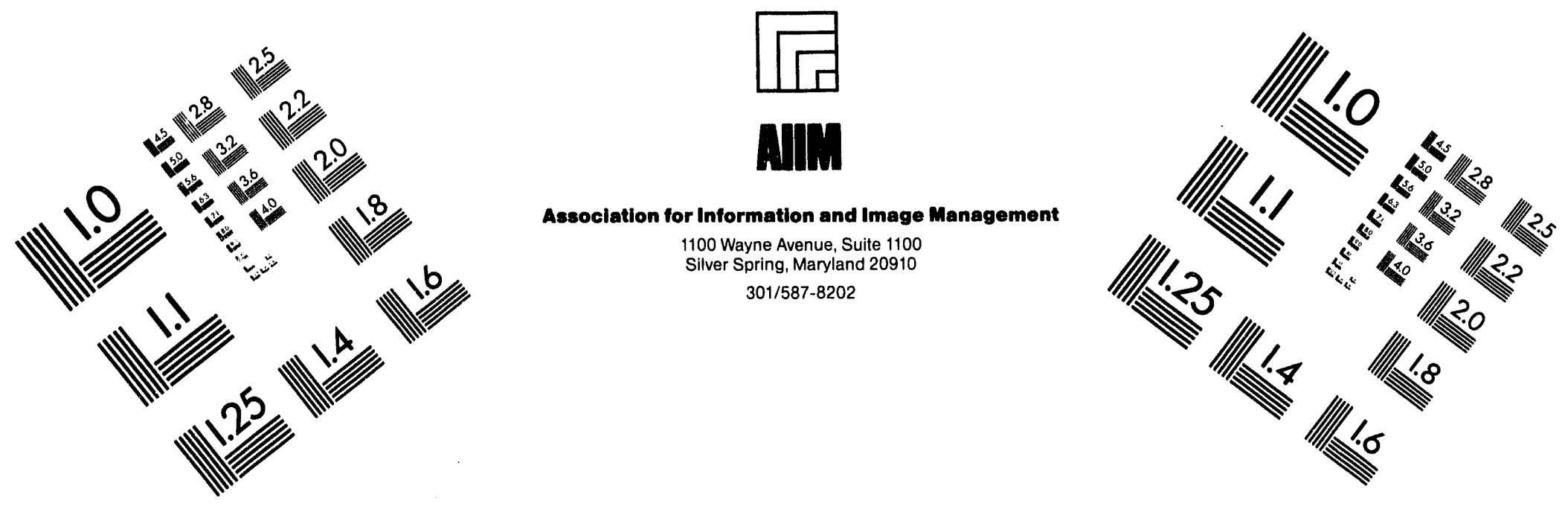

\title{
Centimeter
}

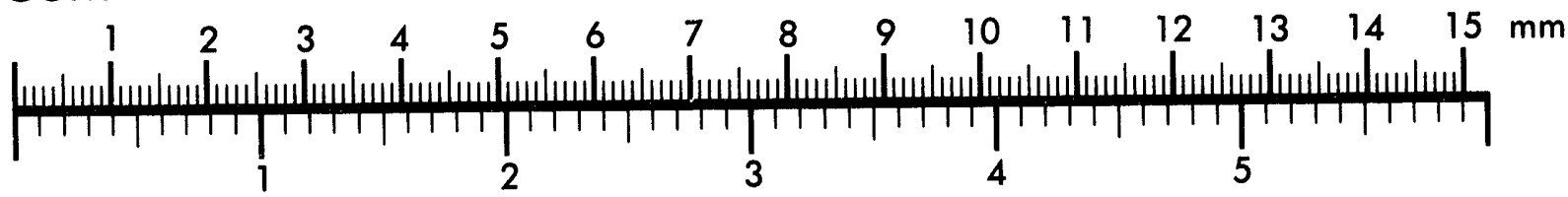

Inches
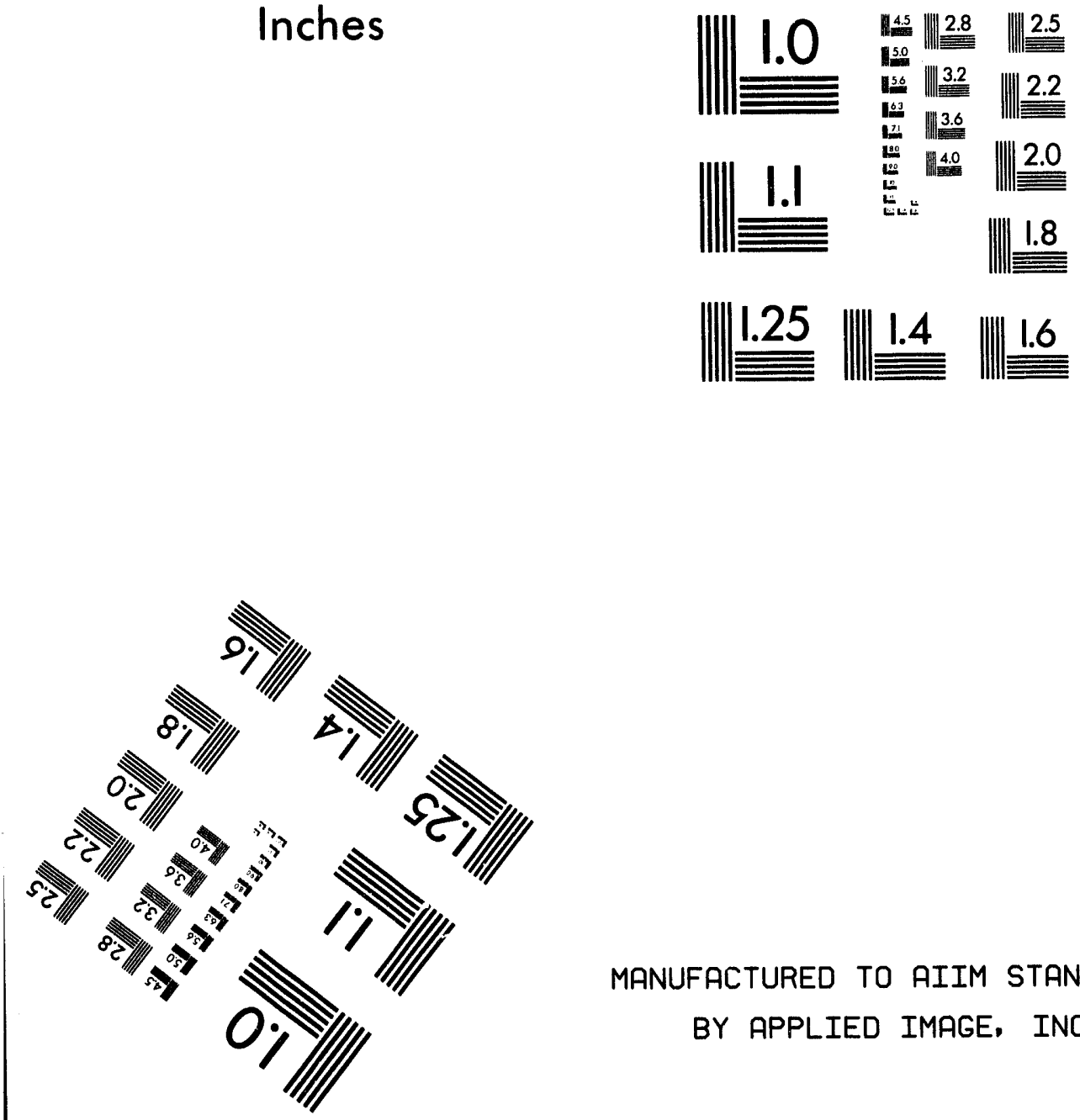

MANUFACTURED TO AIIM STANDARDS

BY APPLIED IMAGE, INC.

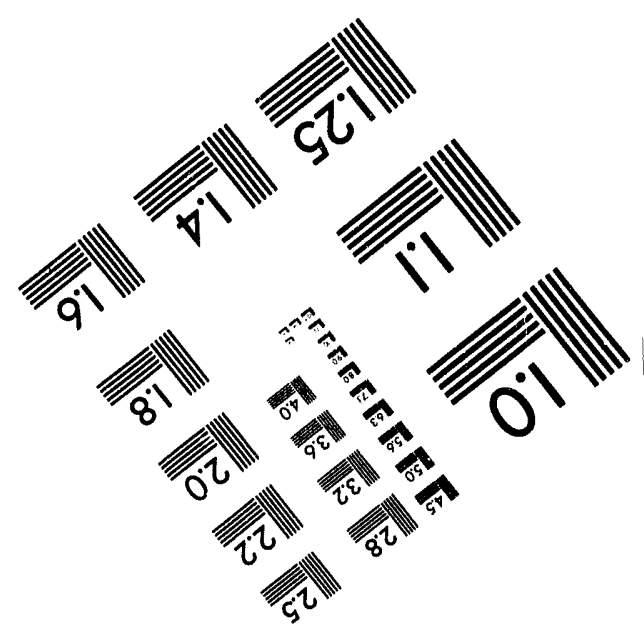



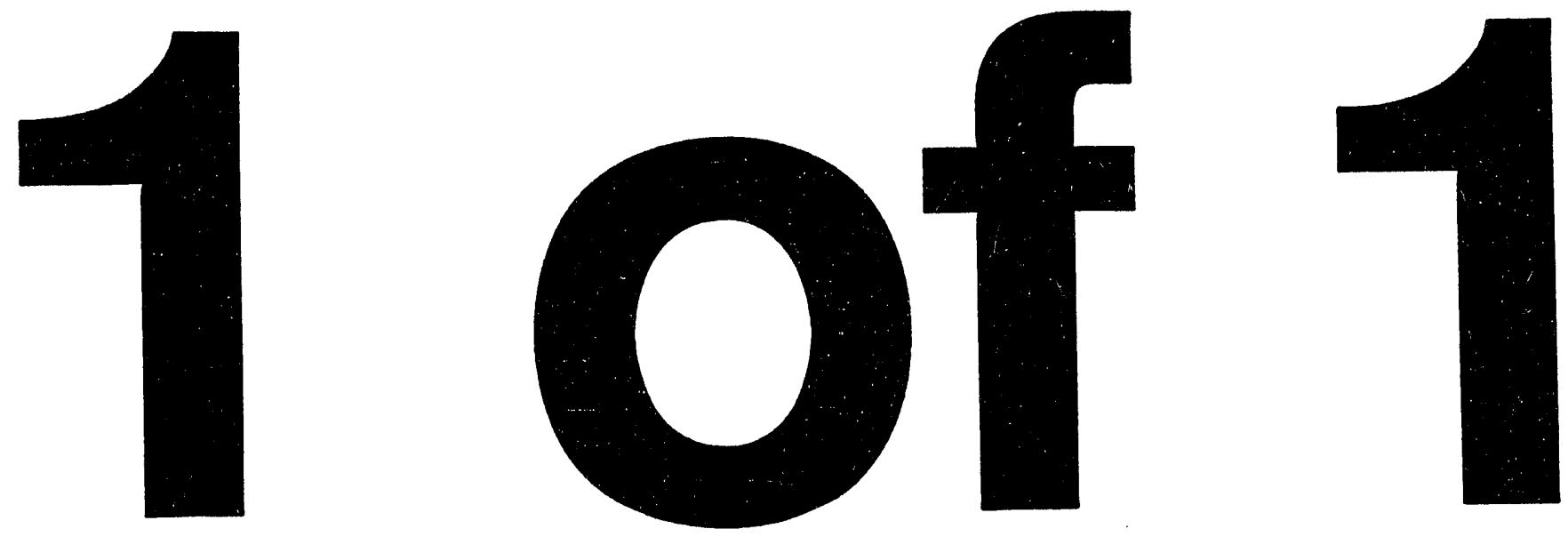


\section{A Medium Resolution Minefield Model Suitable for Entity-Level Resolution Combat Simulations}

Edward T. Powell

June 9, 1994 


\begin{abstract}
A new, flexible, and realistic representation of conventional minefields in entity-level resolution combat simulations is presented. The model includes important aspects of minefield effects on battlefield entities and of breaching devices on minefields. The model is designed at "medium resolution," that is, it is general enough to depict a wide variety of tactical situations accurately; however, it only represents tactically significant aspects of mine warfare, discarding or aggregating details, thus minimizing computer memory and speed requirements. This paper describes the model in detail, its implementation in the Janus simulation code, and its use in a preliminary analysis effort related to the effect of delay on the tactical battlefield.
\end{abstract}




\subsection{INTRODUCTION}

1.1 Motivation. Mines and barriers are a critical part of modern warfare. The use of mines requires careful planning and countermine efforts require precise

execution. Also, to prevent friendly mines from becoming obstacles to friendly forces, even more careful planning is required. Currently, new technology promises a dramatic increase in mine effectiveness. Possibilities for the near future include: smart minefields in which individual mines communicate with each other to determine the nature of the threat and the best method of attack, standoff munitions that are capable of autonomously attacking the enemy from 100 meters away or farther, and increased conventional mine lethality. As the potential aggressors of the world acquire more sophisticated technology, the US will be increasingly faced with high-technology counter-mobility-based threats for which effective countermine capabilities will be needed.

Modeling and Simulation (M\&S) are important tools for use in determining what mine/countermine capabilities are needed. From detailed engineering models of various proposed technologies to large scale corps- or theater-level combat models, M\&S can be used to study questions of importance to decision makers, with the goal of providing timely and accurate information about which technologies best meet a given need, and how new technologies interact with current and future tactics. It is the study of this last issue, the interaction of technology and tactics, that requires a special type of simulation, one that includes all of the essential tactical details of a military operation, without so much aggregation that the effect of any new technology or tactic is "washed out."

To understand the interaction of new mine and barrier technologies with tactics and doctrine, a combat simulation that models the following functional areas is required: movement, direct and indirect fire, mines, barriers, and other engineer 
activity. It must operate at the entity level, modeling individual vehicles and soldiers on the battlefield. The simulation must have some level of semiautomation, which enables the analyst to concentrate on tactical problems rather than simulation-specific minutiae. In an ideal world, a fully automated model could be used, one that includes realistic decision making on the part of the computer generated forces. Unfortunately, such a model does not yet exist. Therefore, interactive simulations are used, with a human in the loop making tactical decisions. To study mobility/countermobility issues in particular, the simulation requires a mine model that includes mine effects, different mine types, minefields with extent and different shapes, multiple types of mines in a single minefield, the effect of breachers on the minefield, real-time deployment of minefields, and the capability to add limited intelligence to the minefields.

There have been a number of simulations used in the past to study military engineering issues. Some large-scale aggregated models have increased the fidelity of their engineer play recently, to allow more precision in studying engineering issues. However, even as these models get more detailed, they still suffer from the problems associated with being aggregated expected-value models of a process (combat) that is essentially stochastic in nature, characterized by numerous probabilistic events. Combat at the tactical level can best be studied using stochastic, entity-level resolution models, with multiple runs yielding a distribution of results. There are a number of simulations that meet this description: CASTFOREM, a non-interactive, decision-table-driven simulation, and the Janusderived interactive simulations, such as Janus ${ }^{1}$, Janus-A, JCM, and UCCATS.

CASTFOREM incorporates the Canadian-developed minefield model called Proca (or AT-MESIM). This model is very detailed, and assesses mine kills and breaching effects depending on the exact geometry of the interaction between the individual mine and the interacting entity. 
Each of the Janus-derived simulations has certain similarities with the others, but there are a number of differences, especially in the mine warfare aspects. Janus and JCM use a very simplistic linear minefield model, in which a unit (composed of a number of entities) would lose one of its entities to any minefield encountered, and (if it had breaching capability) would be delayed a predetermined period before breaching the minefield. Janus-A includes a simple mine model allowing for a small number of different types of mines, with a fixed number of mines per minefield. Mines are laid out in rows in a minefield, and each mine is individually placed on the ground. UCCATS uses a different approach to minefields, modeling them as rectangular fields that have a certain probability of killing any entity that tries to cross. Breach lanes, which persist in time, can be constructed by breaching entities over the minefield.

All of these models have their strong and weak points. The Janus-derived simulations' minefield models suffer from being low-resolution representations, with limited correlation to reality. The most accurate of these models, those in Janus-A and UCCATS, are still too low resolution for accurate mine/countermine analyses. The CASTFOREM high-resolution minefield model is very accurate, but CASTFOREM is limited to about brigade-size battles, and is not interactive. What was required was a model of intermediate (or medium) resolution incorporated into one of the Janus-derived interactive simulations: a model that included most of the tactically important aspects of the high resolution model, but used certain aspects of the low resolution models to enable it to be useful with large scenarios.

The basic idea behind the new medium resolution model is the concept of indeterminate placement of mines in a simulated minefield. That is, minefields are represented as polygonal regions containing a certain density of mines. Only when an entity interacts with a region do the positions of the mines in that region need to be calculated, and then the positions of only those mines that are in the path of the 
entity need to be determined. In mine warfare, only a fraction of those mines laid down on a battlefield are ever encountered. Thus, using indeterminate placement, scenarios can be imagined that use hundreds of thousands of mines but do not require large amounts of computer memory, because only those mines encountered by battlefield entities are given actual positions.

The following sections describe the algorithms used to make the indeterminate placement concept work, the techniques used to integrate the model into the Janus simulation, and a preliminary analysis performed to test and evaluate the mine model in an analytic environment.

\subsection{MODEL DESCRIPTION AND ALGORITHMS}

2.1 Minefield Model. The new conventional minefield and barrier models are designed to be used with each other in a synergistic fashion. Conventional mines are not, in general, used in isolation, but are combined with other countermobility techniques into a complex obstacle. Using the new minefield and barrier models, the representation of complex obstacles becomes possible in Janus simulations.

The models are designed in an object-oriented fashion (shown in Figure 1). A minefield consists primarily of a list of mine regions, but also ircludes an enclosing polygon and rectangle, used for quick determination of whether an entity will encounter a minefield in any given step. The mine region is designed to be an area with uniform properties (such as mine density). The mine densities can be thought of as a series of layers of mines, one on top of the other (see Figure 2). A minefield's regions change dynamically in shape and number as entities interact with it. Associated with each mine region is: a convex polygonal shape, a series of mine types, a series of corresponding mine densities, and potentially a list of individually placed mines. The mine type is an index into a table of different types of mines. 
Associated with each mine type is: a name, and a count of the number of times this type of mine must be triggered before exploding. Individually placed mines occur only under certain circumstances. (See the discussion of indeterminate placement below for a detailed description of these circumstances.) An individually placed mine has associated with it: a mine type, a count of the number of times this particular mine must be triggered before exploding, and an explicit position on the ground.

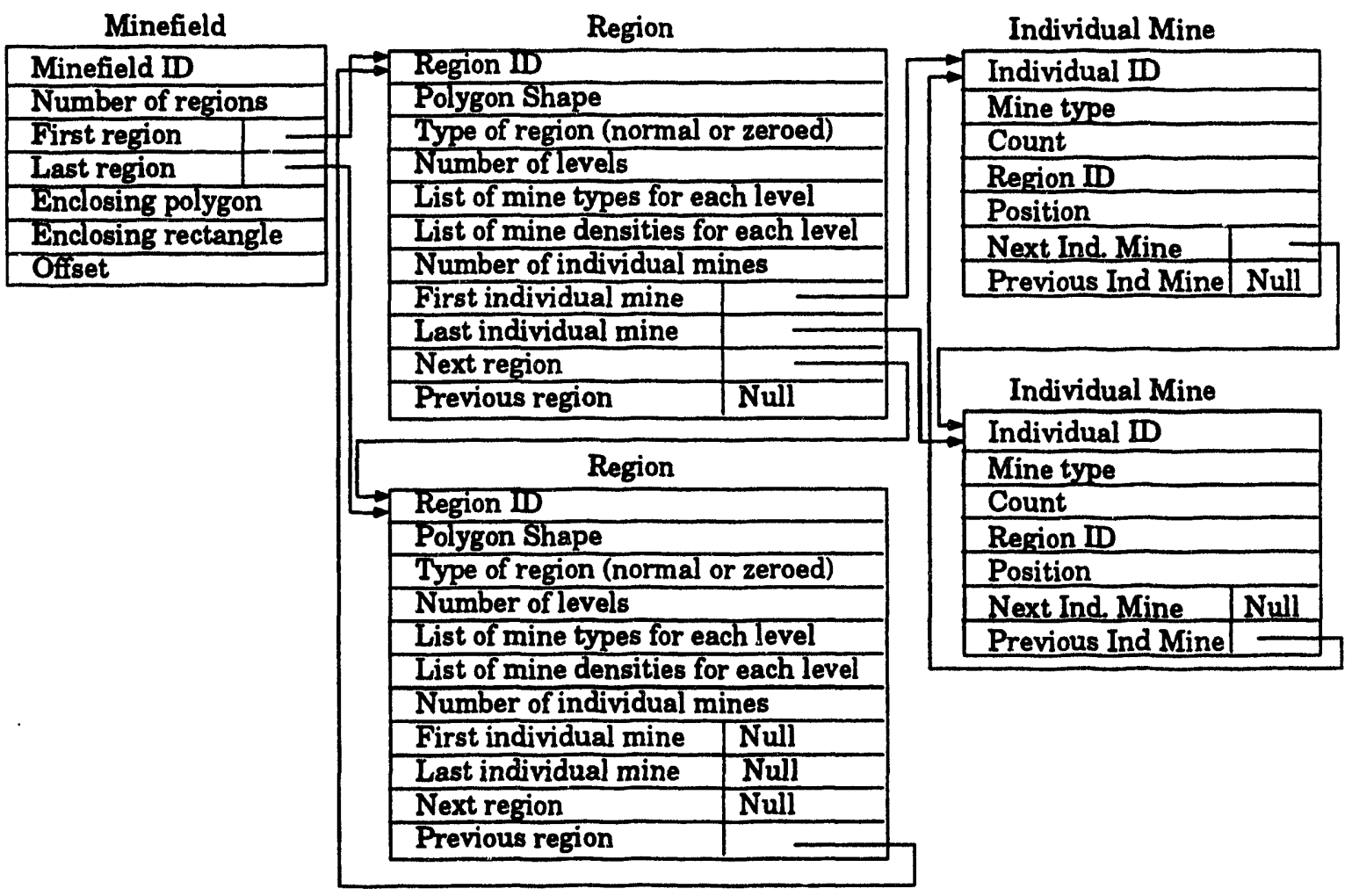

Figure 1: Minefield model data description

Mine Region (need not be rectangular)

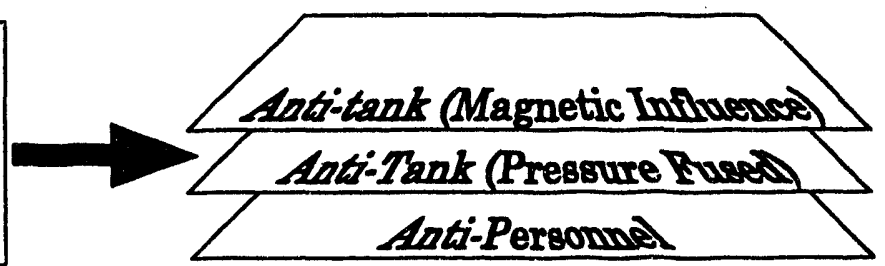

Figure 2: Minefield model layer concept 
Because entities interact with mines and barriers, certain entity parameters must be defined to make the minefield model workable. The primary attribute of an entity in this context is its platform type. The platform represents the physical description of the entity on the battlefield. With the new model, it is possible for an entity to have two different platform types associated with it. Two platform types are necessary to distinguish the mobility and vulnerability characteristics of the platform while in normal operation and also while breaching. For instance, a tank with a plow attached has very different characteristics (maximum speed, for instance) depending on whether the plow is up or down. An entity's primary platform type represents the platform of the entity while it is not breaching (i.e. the tank with plow up), and the secondary platform type represents the platform while breaching (plow down). An entity with no breaching capability uses only its primary platform type. The attributes of a platform that are needed for interaction with a minefield are: the vehicle's length and width, its speed, and a description of its areas of effect. The areas of effect are illustrated in Figure 3. A platform may have up to seven areas of effect depending on how detailed a model the analyst requires. A tank could be modeled with just one area of effect, representing an average over the whole vehicle. Or it could have three areas, representing the two treads and the underside. This formulation gives the analyst maximum flexibility for defining platforms with as much detail as needed, without having to include all of the detail all of the time. 


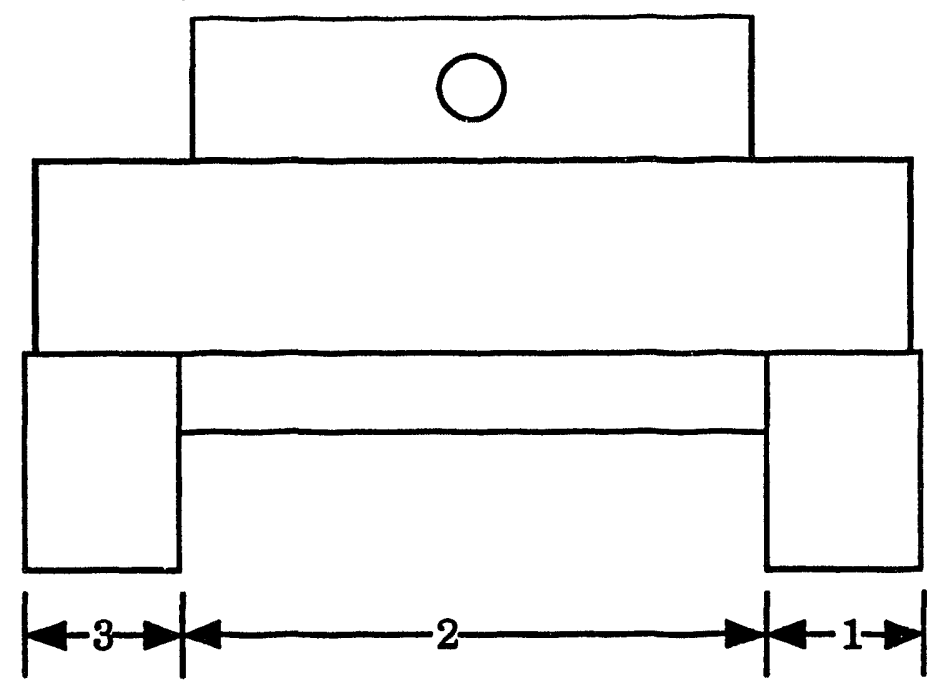

Figure 3: An entity's areas of effect, numbered from the entity's left.

Note that the area of effect represents the effect of the platform on the minefield, not vice versa. Areas of effect are intended as a means to incorporate the different types of breaching mechanisms, not to represent areas of differing vulnerability of the platform to the mines. This feature was designed for computational efficiency, and is (in the context of the current simulation) an adequate approximation to reality, because Janus makes no distinction between mobility kills, firepower kills, and catastrophic kills. This feature, however, could be altered in the future if needed.

To describe the interaction of entities and mines, three tables are needed. The first table contains the probability of a mine being triggered. It has an entry for each mine type, each platform type, and each area of effect for that platform, and represents the probability that a mine of that mine type will be triggered when encountering that particular area of effect on that particular platform. A similar table is required for the probability of the mine being destroyed (or otherwise rendered non-functional) by the entity when encountered. The final table represents the probability that a mine of a particular mine type will kill a particular platform. Because a platform's areas of effect represent the effect of the platform on 
the minefield, this final table lists probabilities of kill for only the platform type and the mine type, and does not include areas of effect.

In the Janus simulation (and in the others derived from it), entities move in discrete steps, the size of the move depending on whether the entity can maintain its desired speed over the terrain. Though the size of the step can be set by the user to any value desired, a default size of approximately $\mathbf{5 0}$ meters is generally used. As the entity steps from one position to the next, it checks to make sure there are no obstacles that would prevent its movement. The new minefield and barrier models rely on this step-wise movement model.

The basic idea behind the algorithm for entity/minefield interaction is shown in Figure 4. (a) An entity approaches a minefield. (b)-(c) It is determined that the entity's path will intersect the minefield in the current step, and an assessment of the minefield's effect on the entity must be made to determine the actual size of the step. (d) Once this determination is made (as well as the assessment of whether the entity was killed or not), and the extent of the entity's movement is known, the effect the entity had on the minefield can be determined. It is very important to note that these two assessments are separated. First the minefields' effect on the entity is determined, then the entity's effect on the minefields. If these two assessments were not separated, it would be very difficult to efficiently account for overlapping minefields. 
Minefield (shade represents mine density)
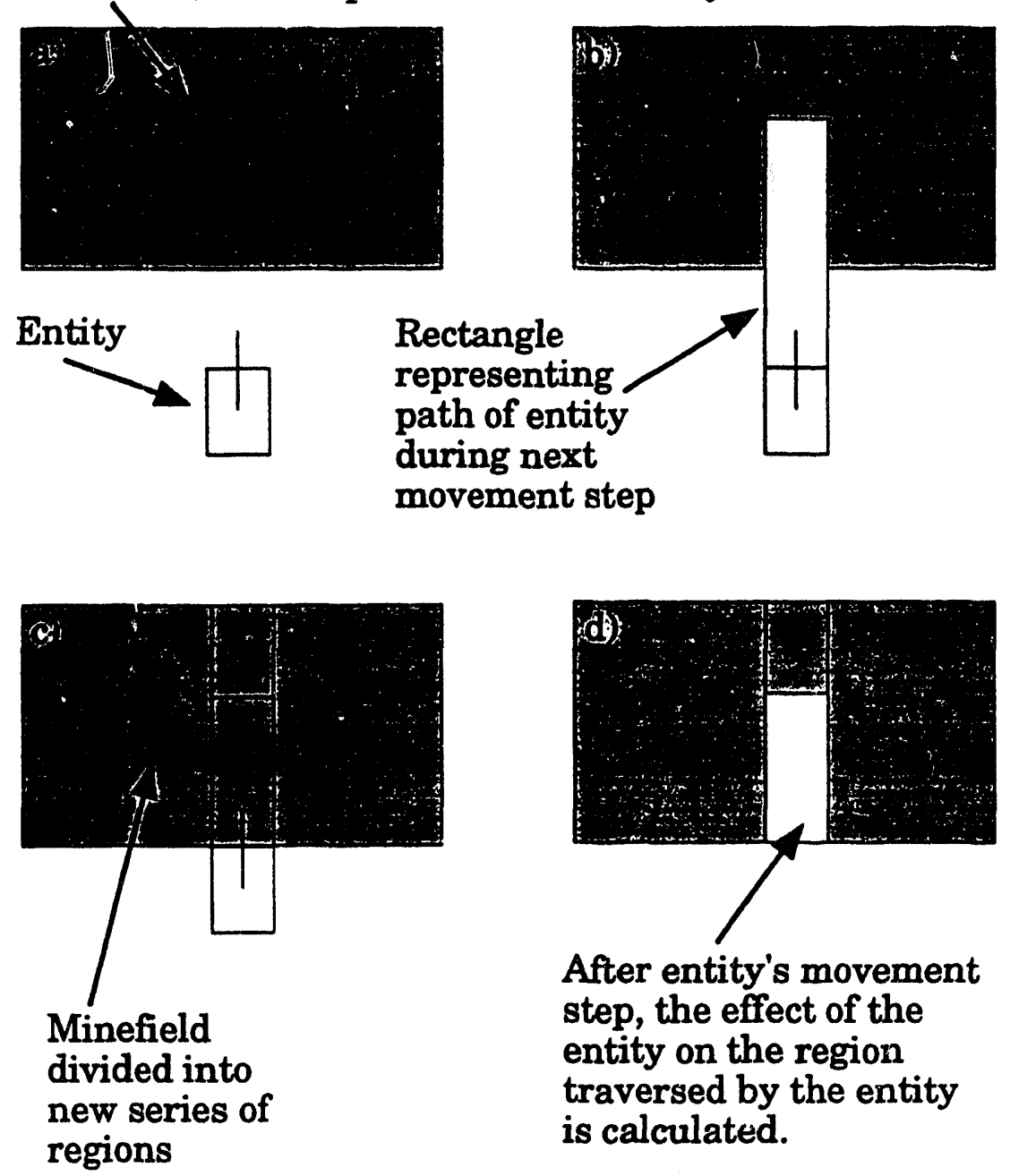

After entity's movement step, the effect of the entity on the region traversed by the entity is calculated.

Figure 4: Interaction of an entity with a minefield. a) The entity approaches a minefield. b) A rectangle representing the entity's next movement step is calculated. c) The entity path rectangle is intersected with the minefield, yielding a number of regions. d) After the effect of the minefield on the entity is calculated, the effect of the entity on the minefield is determined, and the minefield regions are updated accordingly.

Regions traversed by the entity are divided into a number of smaller regions: regions outside the entity's areas of effect (which are unchanged in content), and regions that the entity interacted with (which can be changed in content). Regions that interact with the entity can change in two ways. First, if a region contained individual mines, these mines could have exploded, and thus need to be removed from the region. Second, if the region contained mine density, this density could be 
altered by the entity's passage through the region. As more and more entities or breaching devices interact with the minefield, more and more regions are created. A technique for limiting the proliferation of regions is needed to prevent an exponential explosion in the number of regions. Such a technique is described below.

Mines are only given positions on the ground when needed. Mine densities are used whenever possible. This is the concept of indeterminate placement. When assessing the effect that traversing a mine region has on an entity, that region's mine density is temporarily transformed (i.e. deaggregated) into a list of individually placed mines positioned randomly in the region. The effects of these mines are determined in order of encounter. If any of the mines kill the entity, the entity is said to have died at the position of that mine, the assessment process is stopped, and the length of the entity's movement step is recorded. The sequence of events is summarized in Figure 5 and is expanded upon in the Appendix. Note that the minefield has not been changed in any way so far. Since it is possible for an entity to encounter more than nne minefield in any one movement step, it is necessary to repeat the maximum movement step calculation for each of the minefields that the entity encounters in a step. Then the smallest maximum movement step achieved is said to be the actual movement step. 
For each entity's movement step:

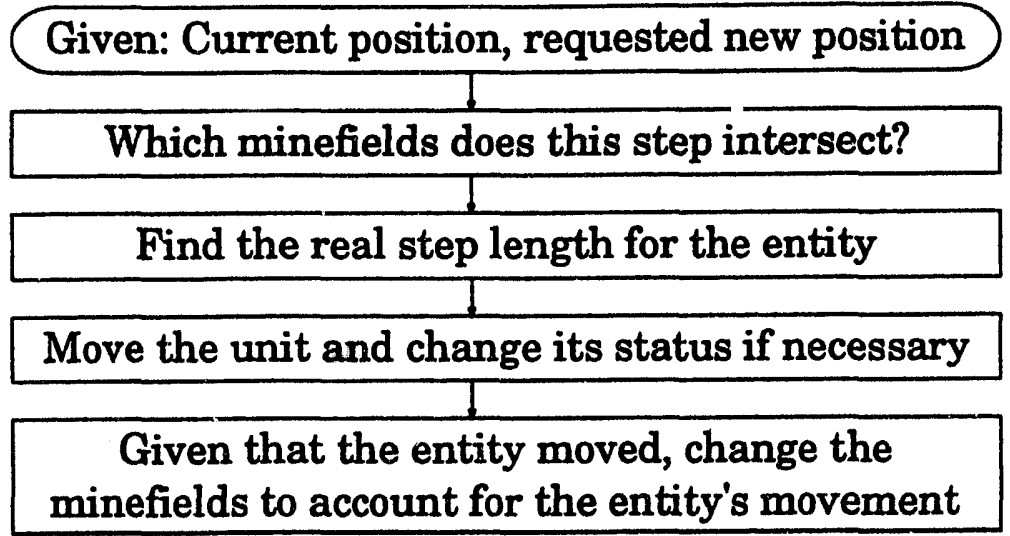

Determining the step length:

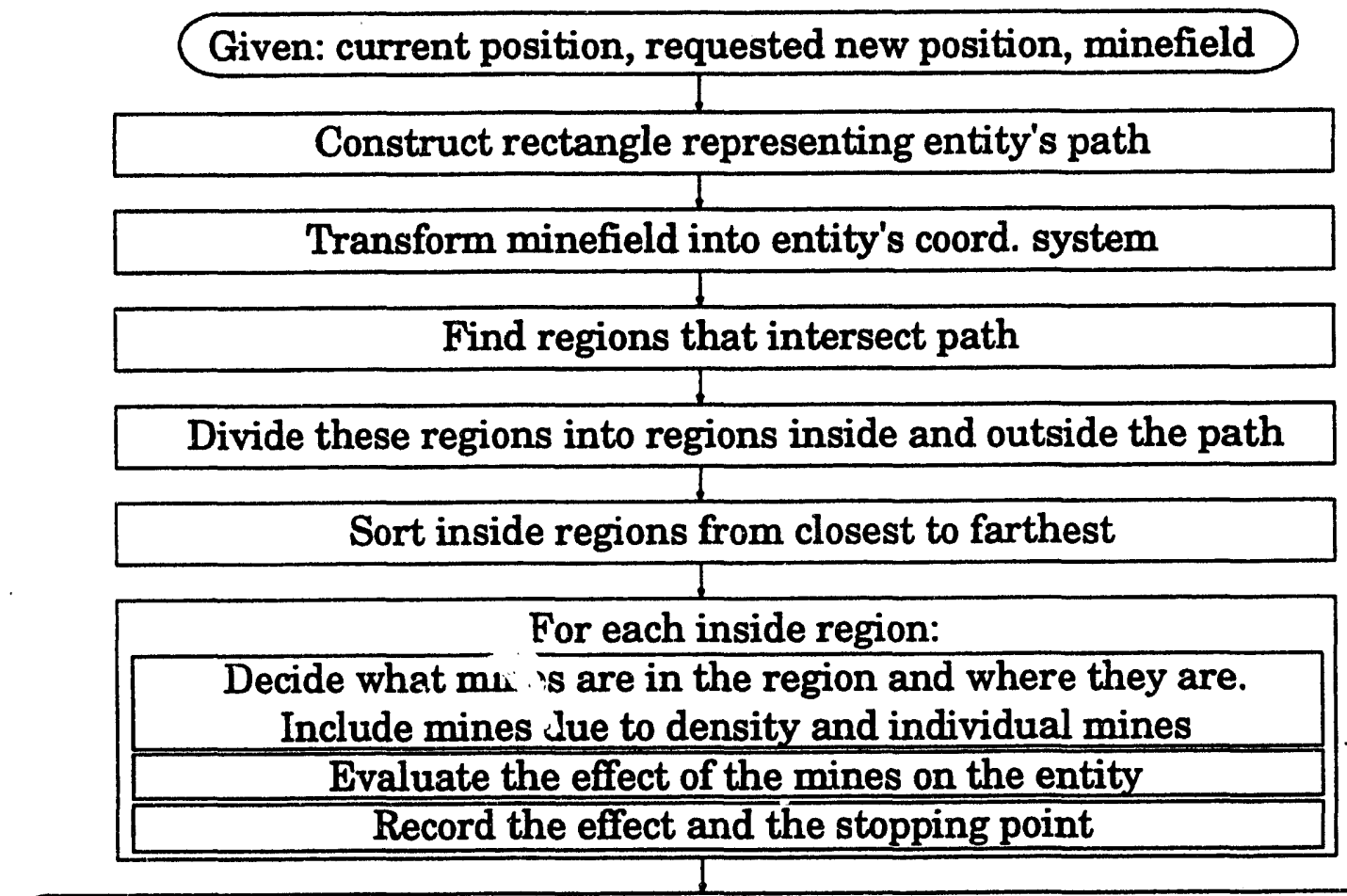

Return the actual stopping point and the effect of the minefield on the entity

Figure 5: Movement and step length logic.

Once the actual movement step is calculated, it is now possible to assess the effect of the entity on the minefield. Each minefield is updated using the knowledge that the entity moved as far as it did. That is, given that the entity moved a certain 
distance (before either being killed or reaching the end of its step), each mine that the entity encountered during the step must have either: (1) been destroyed (or otherwise removed) by the entity, (2) not triggered, or (3) triggered, but did not kill the entity. Though cases (1) and (3) have similar results, i.e. the mine is gone, the difference is that in case (3) the mine explodes (and can thus be detected), whereas in case (1) the mine is destroyed without possibility of detection. Mine densities are adjusted using the table values for the probabilities of each of these possibilities. Individual mines are left in the minefield or removed depending on random numbers drawn against each of the same probabilities.

The use of individual mines in mine regions is an important aspect of the medium resolution model. Individual mines can appear in four basic situations. First, individual mines associated with a minefield region can be defined at the beginning of a scenario. Therefore, it is possible for the user to specify in advance the types and positions of individual mines. Second, if a mine that is designed to explode only after being triggered more than oace is encountered in the assessment calculation while mines usually represented as mine density are deaggregated, that mine is not reaggregated into the mine density. but left in the region as an individual mine. The trigger count of the mine is decreased by one and the mine density (after any other mines are reaggregated) reflects the lack of the mine left as an individual. Third, the user can specify a mode in which any minefield encountered by an entity is immediately and totally deaggregated and left that way for the extent of the simulation. Finally, a region with a low density of mines is automatically deaggregated into a region with no mine density, just individual mines.

It is this last rule, combined with two other rules, that prevents the number of regions from increasing exponentially as more and more entities interact with a minefield. These rules can be stated as follows: (1) If the expected number of mines in a region (that is the mine density times the region's area), becomes less than a 
fixed number $N$, the representation of that region is changed from mine density to individually placed mines. Such a region is said to be zeroed, in that the density has been zeroed out in favor of an individual mine representation. (2) Zeroed regions are never subdivided any further. This rule places an upper limit on the number of regions into which a minefield can be divided. (3) Regions that have neithe: mine density nor individual mines are deleted from the minefield. This rule tends to decrease the number of regions in a minefield. In practice, when these rules are implemented, the number of regions in a typical minefield seldom rises above twenty.

Finally, it is useful to examine the algorithm used to determine the intersection of an arbitrary polygon with the entity path rectangle. This algorithm is the basis for dividing up the minefield into regions traversed by a vehicle and regions not encountered. The algorithm, illustrated in Figure 6, is optimized for the path being rectangular. First, any region which intersects the path rectangle is transformed into the coordinate system in which the path rectangle is vertical with the entity traveling "north." Second, it is "sliced," first to the left of the path, then to the right. Finally, the region is sliced by the trailing edge of the path, and then by the leading edge. Slicing the region in this fashion is very efficient, since a general intersection algorithm need not be developed. Also, since the region is first sliced to the sides of the entity's motion, the number of subsequent polygons encountered in the next step is minimized. (Note that in Figure $4 \mathrm{~d}$, the entity, if it survives, will only traverse one additional region.) 

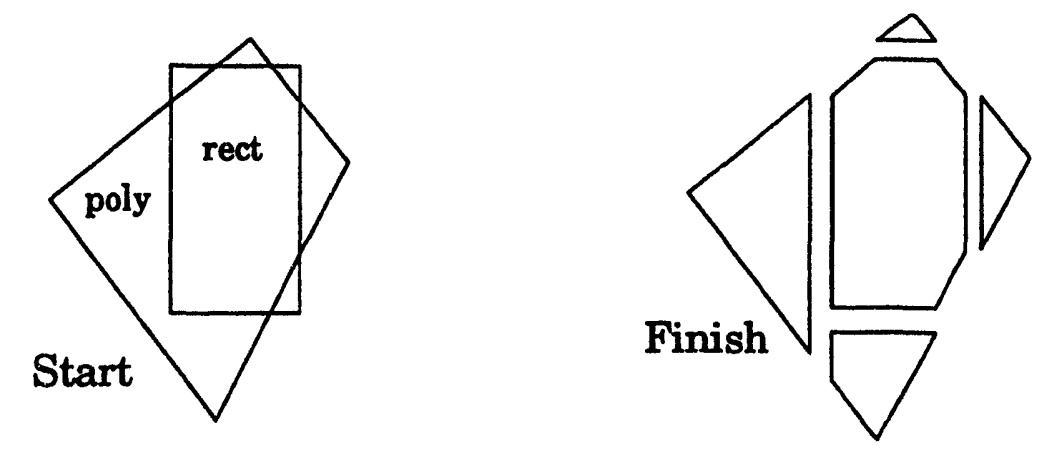

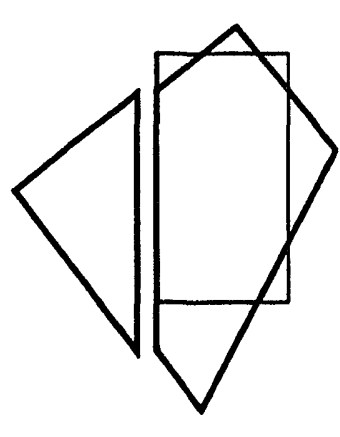

Step 1

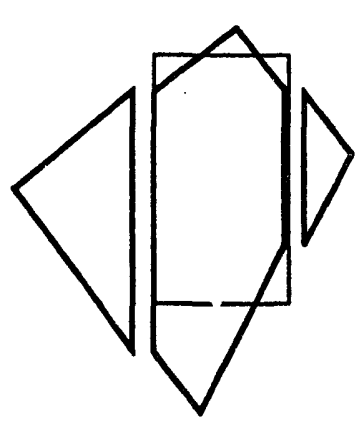

Step 2

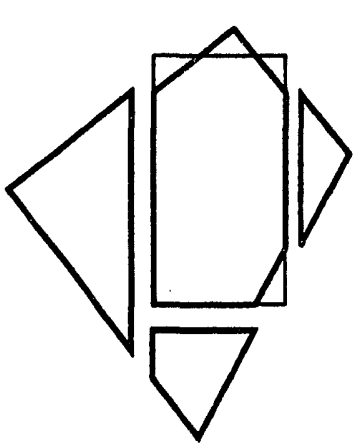

Step 3

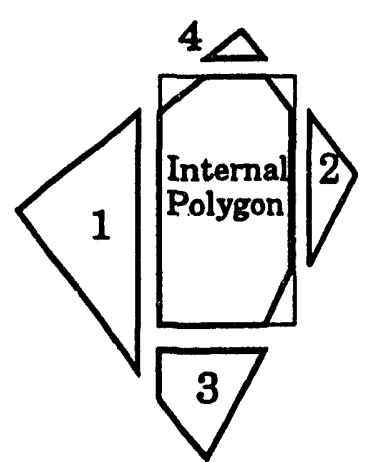

Step 4

Figure 6: Polygon splitting algorithm

In summary, the new minefield model allows the analyst maximum flexibility to define a wide range of different types of minefields and breaching devices. A minefield can be designed with all individual mines laid out in any manner desired. Or a minefield can be defined as a list of regions, each region containing a series of mine types and densities. In the latter case, the mines are laid down only when necessary to evaluate their effects on a passirg entity, or the effect of the entity on the mines. Regions with mine density are used unless the expected number of mines in a region is small, in which case those regions are zeroed out of mine density, and represented as indivisible regions with only individual mines.

2.2 Barrier Model. In keeping with the goal of a medium resolution model, one that does not contain unneeded detail, a one-dimensional barrier model was designed. Barriers are contained in a dynamic list that is updated as they are breached. When an entity approaches a barrier, if it can breach the barrier, it 
begins breaching operations. The breach attempt is recorded in a breach record. The data contents of the barrier and breach records are shown in Figure 7.

Note that the barrier is defined by two endpoints and an offset. The endpoint positions are measured relative to the offset. The offset thus represents a global location of the barrier, allowing the endpoints to be specified with more precision relative to this global position. The barrier's thickness is simply a multiplier that defines the "difficulty" of breaching this particular barrier. The height attribute is intended to allow barriers to block an entity's line of sight (if the barrier can block line of sight). The enclosing rectangle attribute is used for

\begin{tabular}{|l|}
\multicolumn{1}{c|}{ Barrier } \\
\begin{tabular}{|l|}
\hline Barrier ID \\
\hline Barrier type \\
\hline First Endpoint \\
\hline Second Endpoint \\
\hline Barrier Thickness \\
\hline Barrier Height \\
\hline Enclosing rectangle \\
\hline Offset \\
\multicolumn{1}{|c|}{ Breach } \\
\hline Breach ID \\
\hline Barrier ID \\
\hline Entity ID \\
\hline Breach Position \\
\hline Time breach started \\
\hline
\end{tabular}
\end{tabular}

Figure 7: Barrier model data description quick determination of whether an entity will encounter the barrier in any given movement step. A breach is a mechanism for tying a breaching entity to the barrier being breached. For every platform type and barrier type there exist two quantities that define the possible interactions. The first quantity is the time to breach. If this value is negative, the platform is not capable of breaching the barrier. The second value is the width of the breach. All classical barriers, such as berms, ditches, walls, fences, etc., can be described in the same way in this model. Barriers that operate over areas (such as an abatis) can be represented as a series of these one-dimensional barriers.

The operation of the barrier model is illustrated in Figure 8. As an entity approaches the barrier, if it cannot breach the barrier, it halts. If it can breach the barrier, it automatically goes into breach mode. The amount of time required to breach the barrier is the thickness of the particular barrier multiplied by the timeto-breach entry in the barrier interaction table. A breach entry is created for the 
entity and the barrier. An event is scheduled for the end of the breach delay so that the barrier can be updated and the entity can be restarted on

a)

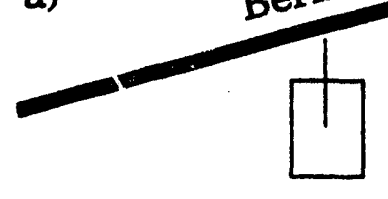
its path.

When the entity completes breaching the barrier, two things occur. First, the barrier is split into two separate barriers, each with its own unique identification number. It should now be clear why a breach record is required. For example, two entities (A and B) are simultaneously breaching the same barrier (barrier 1 ) at different locations along its length. Entity A succeeds in breaching the barrier, which now becomes two barriers, barrier 2 and barrier 3 . (Barrier 1 no longer exists.) However, if no record of the breach is made, the simulation will still believe entity $B$ is breaching the nonexistent

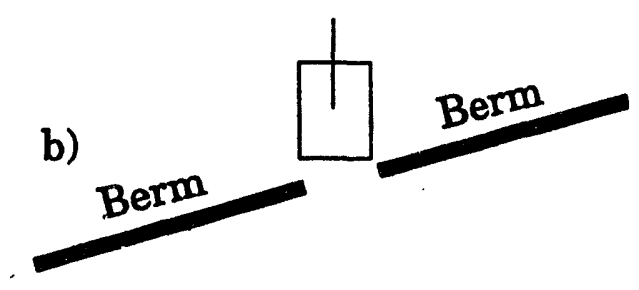
barrier 1. Therefore, the second action taken

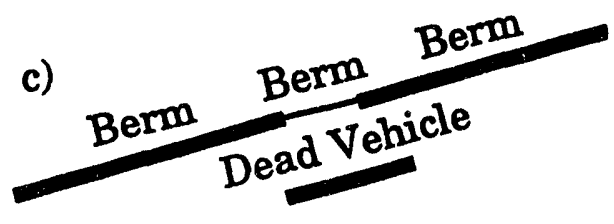

Figure 8: Barrier Model. a) An entity approaches a barrier. b) After a breach time, the barrier is divided into two barriers. c) If an entity is killed while breaching, a partial breach is made, and a dead vehicle barrier is placed where the entity died.

when a breach occurs is to look over the list of breaches currently pending, find any associated with the former barrier (1) and replace the entry in the breach record with the proper (new) barrier identification number. In the current example, perhaps entity $B$ will now be continuing to breach the new barrier 2 . In this fashion, a fully dynamic barrier model can be created, and multiple entities can breach a barrier simultaneously. It should be noted that in the current implementation multiple entities cannot help each other on the same breach.

Finally, Figure $8 \mathrm{c}$ shows what happens when an entity is breaching a barrier and is killed. First, a partial breach of the barrier is made, depending on the 
amount of time the unit was breaching the barrier. Second, the dead entity is transformed into a new barrier, a dead vehicle barrier, at the position occupied by the entity when it was killed. Thus four barriers with differing attributes replace the original barrier.

In summary, the barrier model has been created at medium resolution, its one-dimensional nature simplifying greatly the computational burden, while retaining most of the important tactical details required for analysis of mobility/countermobility issues.

2.3 Janus Integration Issues. The minefield and barrier models were designed, tested, and implemented in a testbed simulation. This development method was chosen so that the model could be developed independent of any particular simulation, and in principle could be inserted into any entity-level simulation. For the purposes of evaluation and analysis, the minefield model was first implemented in Janus. Because this code was the first one that used the new minefield model, it is important to list the issues raised by incorporating the model into a specific simulation.

Janus is supported with various specialized data editors and post-run analysis tools. Graphics terminals are used to display the terrain and the simulated activities such as movement and engagement. Integrating the new minefield model involved making changes in the movement algorithm, the display of simulated activities, and the data input and output. The integration effort had two constraints: It had to be done in a reasonable time period, and the new code had to be able to read all of the same input files that the unmodified code could read.

In Janus's previous minefield model, breaching was modeled as an activity accomplished over a period of time without movement. In the new model, an entity can move some distance into the minefield without setting off a mine, even if it has no breaching capability. To accommodate the new minefield model, additional data 
structures and movement logic were developed. The additional data structures contain all of the necessary minefield data shown in Figure 1. The added movement logic is only used to model movement through minefields. The original minefield model is bypassed.

With the original Janus minefield model, an entity had two options when it encountered an unbreached minefield: eit ${ }^{2}-$ breach the minefield if it was capable of breaching, or halt. With the newi minef .... nodel an entity must be able to move through the minefield without being in breach mode, if the entity decides (or is ordered) to "bull" through a minefield. Therefore, Janus now allows the player to issue the following movement commands to the entities under his control: unconditionally move in breach mode, move in breach mode as required, and do not use breach mode while moving. These commands are implemented by adding new options to the Movement submenu. The player chooses one of the options, then picks the entity for which the option applies. Each entity is constrained by exactly one of the options at any one time, and the latest assigned choice is the option that applies. The "forced" option causes the entity to move into breach mode. The "auto" option causes the entity to move into breach mode only if it has acquired a minefield. The "no" option will prevent the entity from entering breach mode until a player assigns it another option.

Acquisition of a minefield is based on the distance between the entity and the minefield, illustrated in Figure 9. The value $P_{1}$ represents the probability that the entity has realized that it is in a minefield when

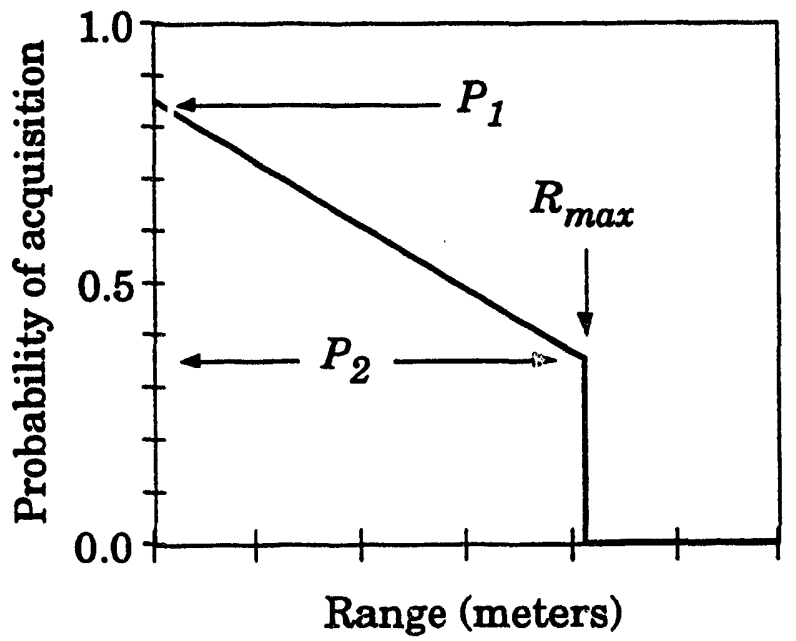

Figure 9: Simple model for the acquisition of minefields. 
it actually is. The value $P_{2}$ represents the probability that it has acquired the minefield at the distance equal to $R_{\max }$, the maximum acquisition range. When a minefield is acquired by an entity in auto-breach mode, the entity immediately goes into breach mode. For distances between zero and $R_{\max }$, the corresponding probability is a linear interpolation between $P_{1}$ and $P_{2}$. For distances greater than $R_{\text {max }}$, an entity will not acquire the minefield. At each movement step while an entity is near a minefield, a random draw is compared to the probability of acquisition of that minefield. If the random draw is less than the probability, the entity acquires the minefield; otherwise, it does not. Currently, the values of $P_{1}$ and $P_{2}$ are global and apply to all minefields equally. In principle, these values should be attributes of mine types, so that each minefield would inherit its acquisition probabilities from the mines that comprise it.

There were some changes made in Janus to the graphics display of the interaction between minefields and entities. Minefield ownership is specified by side and by task force. A special symbol is now drawn whenever an entity acquires a minefield that it does not own. A user can therefore put an entity into breach mode (for its own protection) and scout around the area to determine the probable extent of the minefield.

Another change deals with the display of exploding mines. In the old model, every explosion caused a kill. In the new model, mines can explode without causing a kill. A "mine explosion" symbol is now used to designate when a mine detonates. When a mine kill occurs, it is designated with the usual Janus symbol for a mine kill: V.

The improved minefield model required additional data to function with Janus. All of the minefield-specific data is currently stored in files separate from the regular Janus data files. These minefield data files were constructed so that a standard text editor may be used to alter them. Currently, none of the regular 
Janus data editors can be used to change the new data files. Though the new minefield model was designed to be used with any entity-level simulation, it can be seen that a number of issues (such as graphics represen`ation and data editing capabilities) need to be worked through to get a truly integrated model.

To summarize, a need existed for a minefield model that retains enough fidelity to perform tactically meaningful analyses, but does not clutter the simulation with a lot of unnecessary detail: a medium resolution model. A model that fits these criteria has been developed independent of any single entity-level combat simulation. In this model, minefields are represented as polygonal regions of mine density, and individual mines are positioned only when necessary in a technique known as indeterminate placement. Efficient algorithms have been developed to manipulate these regions. This model has been integrated with the Janus combat simulation, and a short evaluation analysis has been performed, which will be described below. In addition, a new barrier model has been developed, allowing the use of complex obstacles in simulations. The barrier model is not yet integrated into the Janus code.

\subsection{TeST AND Evaluation ANALYSIS: ThE EFFECT OF DELAY.}

3.1 Introduction. To test the minefield model in an analytic context, a study was designed to examine the effect of mine-induced delay on the outcome of a battle. The idea for this study emerged from the notion that mines have historically been thought of as purely defensive weapons. However, there is no reason not to envision future uses of mines in offensive roles as well as defensive ones. Current Army doctrine says that minefields should be used to stop, delay, disrupt, or turn an enemy force. This study focused on the effect of delaying the enemy. To improve the chances for victory, one must ask: how much delay is needed, and what type of tactics and munitions are best for achieving that delay? This study does not seek to 
answer these questions immediately or in general, but to provide a starting point to examine important issues, and to suggest further research.

Similarly, the study was designed to exercise the minefield model in a fairly complex analytic environment to see how well it performed, and to deternine what improvements, refinements, and additions to the model would be needed for further analyses.

Before discussing the details of the scenario and the results, it is important to discuss the analytic limitations that were present during this study. First, the analysis was not meant to be comprehensive. The outcomes of almost all battles are sensitive to the specific attributes of the scenario: the terrain, the force organizations and deployments, etc. To a lesser extent, the outcomes are dependent upon characteristics of the simulations themselves that may or may not have analogs in reality. For instance, there are certain aspects of combat (such as fatigue or fear) that are simply not modeled by the simulations. Scenarios in which real forces would rely heavily on these non-modeled aspects of combat are almost impossible to simulate with any degree of accuracy. Nevertheless, it is possible to design a scenario in which these simulation-specific idiosyncrasies are minimized if not eliminated. It is with this general caveat in mind that the reader is urged to judge the results and conclusions of this short exercise.

Other caveats dealing with the operation of the minefield model in the simulation are required. The minefield model was designed to be used in an interactive fashion, as most Janus-based analyses are performed this way. Because Janus is a stochastic simulation, it is necessary to repeat the simulation many times to accumulate good statistics to get a reasonable grasp of the distribution of results. In general, ten to twenty runs are required for each scenario. Minefield breaching is a very complex task requiring the coordination of numerous independent actions. Because of this aspect of mine warfare, the number of interactive operators required 
for a detailed comprehensive study is at least three. However, this study was not meant to be comprehensive, so certain tactical details have been omitted to allow Janus to be used in a "batch" mode of operation. In batch mode, all of the tactics (via movement orders) are preprogrammed into the simulation, and the battlefield entities follow these movement orders without deviation. This allows numerous replications to be performed in a short amount of time without requiring large numbers of human participants. The problem with this approach is that entities do not react or adapt to changing situations; they merely obey their orders to the end. This drawback, however, was not thought to be critical to the current limited investigation.

In light of the above, detailed breaching tactics were not implemented. Again, this was a simplification to allow batch mode operation. In the simulations, the entities were in auto-breach mode all the time. When encountering a conventional minefield, entities automatically engage any breaching capability, slow down, and move through the minefield. When they no longer detect the minefield (using the minefield acquisition model described above), they exit breach mode and resume normal speed. Thus, all entities bulled through any minefields they encountered, and no deliberate breaches were made. (A deliberate breach would have increased any delay, while decreasing casualties-a topic for further study.)

It was thought useful to compare the use of conventional mines to at least one other munition. The Wide Area Mine (WAM) was chosen for this comparison. A low resolution WAM model was developed in the context of the current capabilities of the Janus code. In this model, when an entity encounters a WAM, a simple probability of kill is calculated from engineering data provided by the WAM contractor (Textron) that includes the probability of detection, firing, hitting, and then killing the target. The WAM's complex acoustic and seismic detection system was represented with a simple range-dependent probabilistic detection algorithm. 
WAMs are assumed to acquire targets at distances of less than 100 meters. WAMs themselves can be acquired visually by enemy entities; however, being small and camouflaged, the probability of acquiring the WAM is quite small and only reaches appreciable levels at distances less than 25 meters.

The breaching tactics used during encounters with WAMs were similar to those used with conventional minefields. To improve their chance of acquiring and destroying the WAMs before the WAMs can fire, entities under WAM attack slow down. When no WAMs have been encountered recently, the entities resume their normal speed.

3.2 Scenario Description. The tactical situation is shown in Figure 10. The terrain is located in North Korea some 20 kilometers north of the Demilitarized Zone. A US (blue) mechanized battalion task force is attacking north with the goal of seizing two crossroads labeled Objectives A and B. A North Korean (DPRK, red) infantry battalion is defending. Two DPRK tank battalions, located north of the mountainous terrain in the center of the figure, have the mission of reinforcing the defending infantry. To do so, they must use two avenues of approach through the mountains to link up with the defenders. To delay the reinforcements, four areas are identified for the placement of mines. 


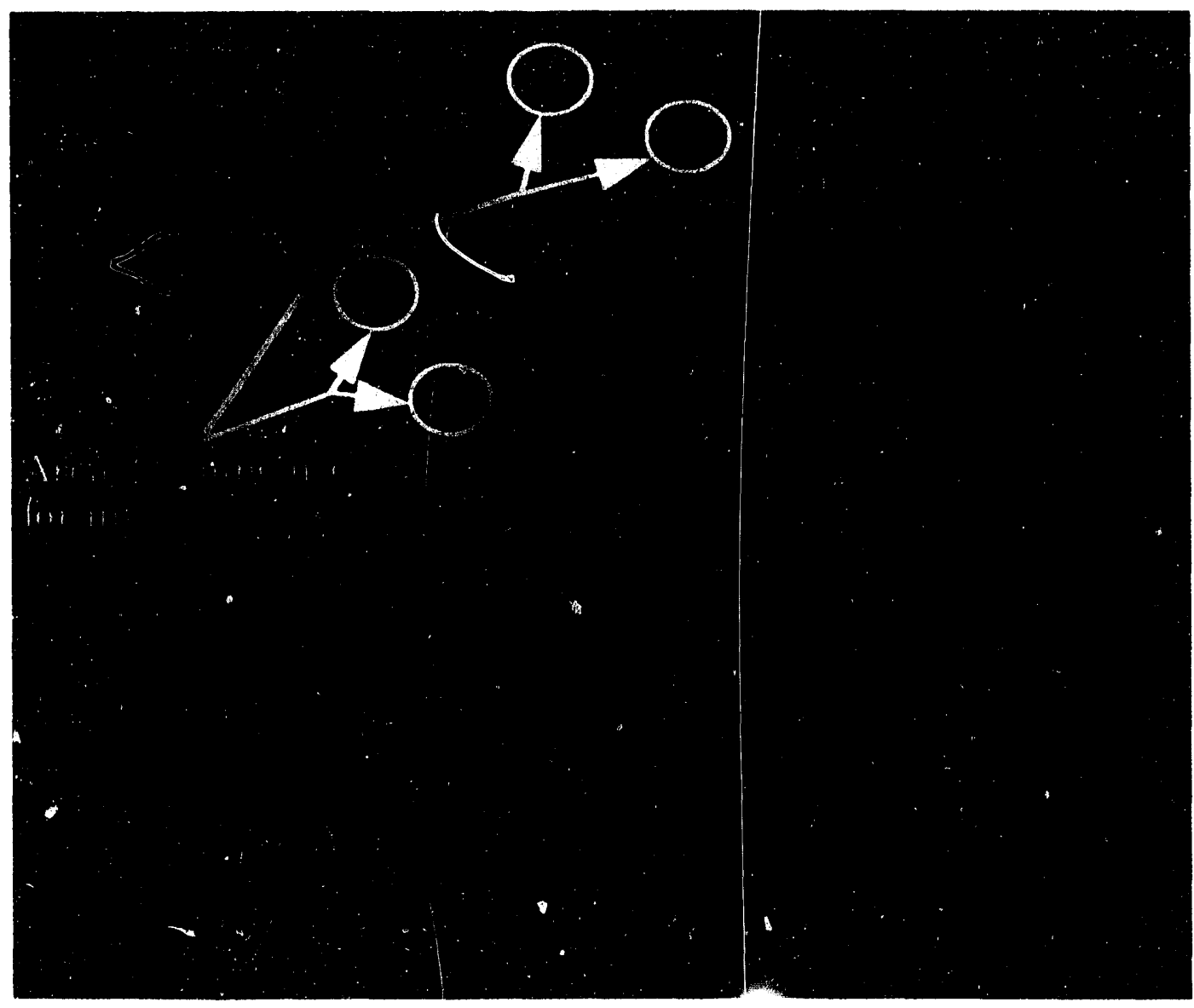

Figure 10: Scenario setup: blue mechanized battalion at lower right plans a twoprong attack on the defending red infantry battalion. Two red tank battalions plan reinforcement; however, four areas have been selected for mine use to delay the red reinforcements

The IS force consists of two tank companies of 14 M1A1 Abrams tanks each, two mechanized infantry companies of 13 M2A.1 Bradley Fighting Vehicles with associated infantry in each company, a scout platoon of $6 \mathrm{M} 3 \mathrm{~A} 1 \mathrm{~s}, 12$ TOW-II antitank teams spread among the infantry companies, and a mortar section of six mortars. The US force attacks on two axes, with the infantry attacking and tanks providing direct support. The mortars have the mission of defense suppression by placing a rolling barrage in front of the attacking maneuver forces. 
The DPRK forces consist of three companies of infantry, deployed in echelon with two companies forward, one back. Within each forward company, the platoons are also deployed two up and one back. Each infantry platoon consists of three squads of six riflemen (each rifleman armed with an automatic rifle and one RPG-18 anti-tank rocket), a machine gun team, and an anti-tank team (armed with an automatic rifle and a reloadable RPG-16 anti-tank rocket launcher). The DPRK infantry is deployed in defilade so as to take maximum advantage of the terrain for cover, concealment, and visibility. The infantry is supported by four BMP-2 infantry fighting vehicles armed with AT-4 anti-tank missile launchers, and nine $82 \mathrm{~mm}$ mortars, whose mission is to suppress and destroy any US maneuver forces. The two reinforcing tank battalions consist of three companies of ten T-72 tanks each plus one headquarters tank for a total of $31 \mathrm{~T}-72 \mathrm{~s}$ per battalion.

Four different scenarios were examined to study the effect of delay. In the first scenario, called the baseline scenario, no mines were used to interdict the reinforcements. In the second scenario, the no reinforcement scenario, the reinforcing tank battalions were not permitted to cross into the battle zone at all. These two cases effectively bracket the effect of mines in blocking the reinforcements. If the mines had no value, the best red could do is the baseline scenario. If the mines were completely effective at destroying the red reinforcements, the no reinforcement scenario would result. Thus these two scenarios give a scale on which to measure the mine performance.

The third scenario used four gauntlets of WAMs in the four areas shown in Figure 10. The concept of a WAM gauntlet consists of scattering WAMs 100 meters apart along an avenue of approach. In this fashion, as the enemy moves along the road, they are under continuous threat of WAM attack. Four groups of 20 WAMs each are pre-placed at the beginning of the game consistent with the use of two helicopters for deployment via Volcano. In the fourth scenario, eight minefields of 
80 anti-tank mines each are used. Two minefields are positioned across the road about 300 meters apart in each of the circled areas depicted in Figure 10. The minefields are represented as a uniform density of mines in a rectangular area with 0.5 mines per meter of front. These numbers of mines are consistent with the use of four helicopters (one helicopter in each interdiction area) using Volcano. In this scenario, air defense threats against the helicopters delivering the mines were not modeled. This study was focused on mine effectiveness, not deployment schemes. As long as a plausible deployment mode exists, the question of the exact deployment mechanics can be determined after the mines are found to be effective in their delaying role.

3.3 Analysis Results. In each of the scenarios studied, the blue forces traveled inside their vehicles to within about a kilometer of the defending red infantry. The blue infantry along with the AT teams then dismounted and attacked on foot, the tanks following close behind providing fire support. The blue mortars provided some suppression against the red infantry positions. The blue tanks and IF'Vs were attacked by the few long range assets available to the red defenders, AT4 anti-tank rockets mounted on four red BMP-2s concealed along the tree line. At this point, the scenario outcomes diverged.

In the baseline scenario, the red reinforcements, having no trouble crossing the mountains, arrived at the battle scene just as the blue forces dismounted and began their attack (about 10 minutes into the scenario). The red tanks had time to take up positions of relative tactical strength before the blue forces became fully engaged in the battle. As blue continued attacking, they were met by fire from the red tanks. Most blue armored vehicles were destroyed by this fire before the blue infantry even began to engage the red positions. Without armor providing support, the blue infantry was essentially destroyed by the red infantry. One hour into the attack, the blue forces essentially ceased to exist. The key point to note from the 
baseline scenario was that the reinforcing red tanks arrived in time to take up effective fighting positions from which they successfully engaged the blue aitackers.

The second scenario had no red reinforcements to back up the defending red infantry. With no tanks to support them, the red infantry had very limited capability to fight the blue tanks and IFVs, and they were defeated handily in the same time frame as in the baseline scenario: 60 minutes. Blue attained both objectives with significant forces, although blue did take heavy casualties doing so.

In the third scenario, WAM gauntlets were used to delay the red tanks. In this case, blue always won, as in the no reinforcement case, but the outcome was much closer and the victory more costly. The WAMs not only killed red tanks, but also caused them to slow down (causing delay).

In the fourth (conventional mine) scenario, blue achieved its objective with some of its forces most of the time. Blue losses were similar in this case as in the WAM case, but red losses were less severe in this case than in the WAM case.

Figure 11 shows the mean red and blue drawdowns, averaged over 21 replications, as a function of time for all four scenarios. The uncertainties (standard deviations of the mean) in the mean blue drawdowns never exceed $3 \%$, while the uncertainties in the red drawdowns do not exceed $1.1 \%$.

Red takes more casualties as a percentage in the WAM gauntlet case (when some red tanks arrive at the battle) than in the no reinforcement case (when no red tanks arrive at the battle). This counterintuitive result is caused by calculating the drawdowns in the no reinforcement case as the percentage of surviving red systems, excluding the tank battalions (since they are not in the scenario). Therefore, it looks as if there are more surviving red systems in the (weaker to red) no reinforcement case than in the (stronger to red) WAM case. In fact red has fewer surviving systems on average (62 out of 251) in the no reinforcement case than in the WAM case (68 out of 313). Nevertheless, red loses badly in both cases. 

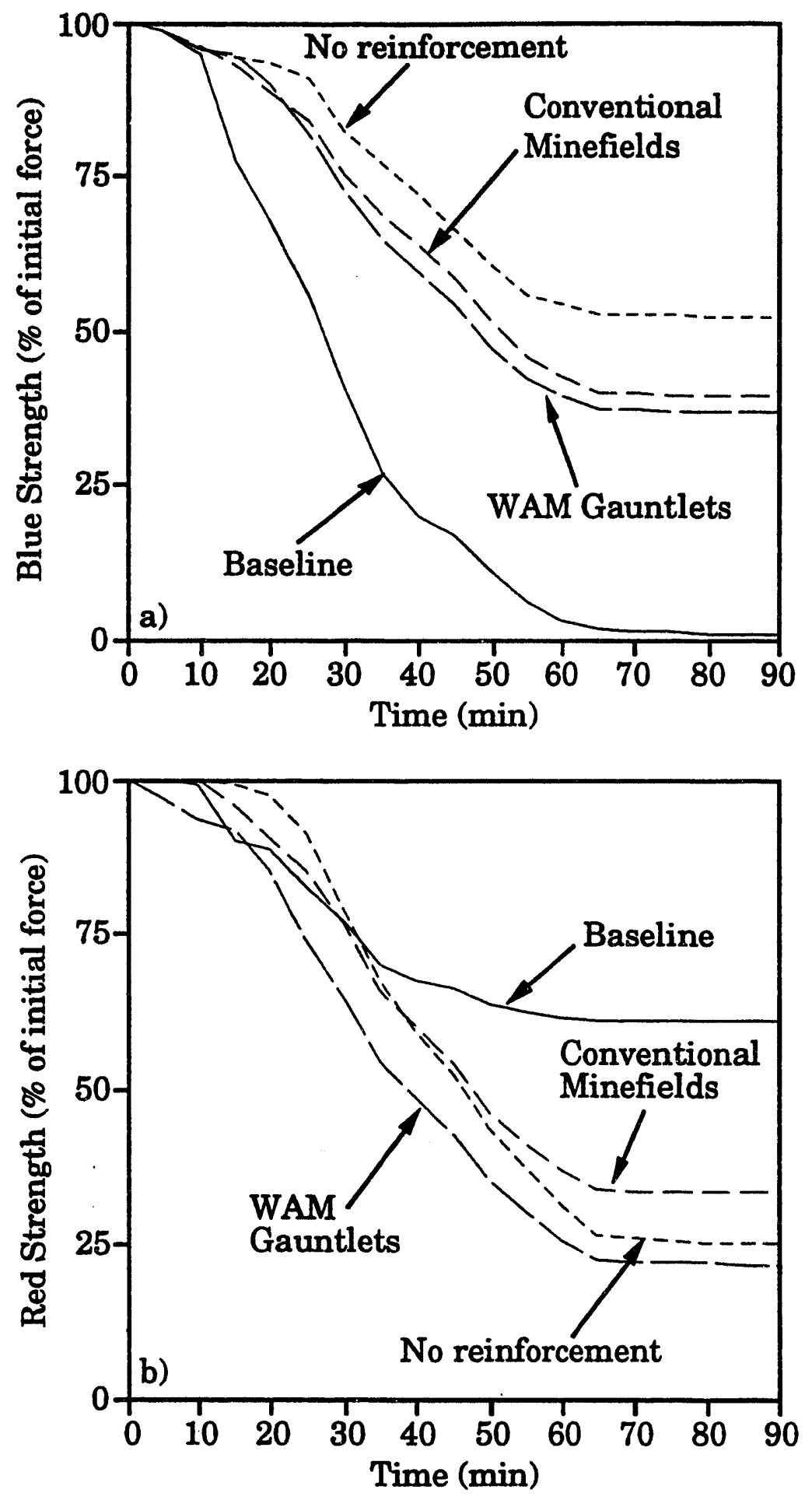

Figure 11: Mean drawdowns (averaged over 21 replications each) for a) blue and b) red force's for all four scenarios. 
The blue side takes substantially more casualties (145 out of 230 systems) in the WAM case than in the no reinforcement case (110 out of 230). The reason for this difference is that some red tanks survived the WAM gauntlet to cause blue some difficulty, although not decisively. The red forces were both delayed and dispersed by the WAM gauntlets. The WAMs caused the red forces to become spread out along the approach roads because the WAMs were spread out over a fairly long distance. Because the red forces were dispersed, they arrived piecemeal to the battle area, and could be destroyed by the blue forces in detail without becoming a major factor in the outcome of the battle. However, because in a real battle a commander might take time to regroup his forces, thus trading dispersal for delay, this dispersal effect may be an artifact of the method of running the simulation in batch mode instead of interactively. The question of trading dispersal for delay must be addressed in future studies.

Blue achieves its objective with some of its forces most of the time in the conventional mine case, with both blue and red suffering fewer losses in this case than in the WAM case. This result is attributed to the type of delay the conventional minefields provided. Conventional minefields caused less delay than WAMs because conventional minefields are much smaller in extent and thus cause the red tanks to proceed cautiously for less total time. Also, instead of dispersing the red tanks as in the WAM case, the conventional minefields tended to bunch up the red forces due to the conventional minefields' limited size on the battlefield compared to the spread out WAM gauntlet. Since the conventional minefields were not as spread out as the WAM gauntlets, the natural reaction of slowing down and entering breach mode was confined to very small geographic areas when encountering conventional minefields, instead of occurring over a long stretch of road (as with WAM). This effect has two consequences. First, the delay allowed the blue forces to attain a commanding tactical situation, and was the cause of the blue 
victory. Second, because the red forces arrived at the scene of battle more or less in a group, blue was less effective at defeating the mass of red forces, leading to a greater number of red survivors overall. On the surface, this result looks questionable. After all, the red tanks should be more effective arriving in mass than piecemeal. This is true. However, the conventional minefields killed more red tanks than did the WAM. (Over half the tanks were killed trying to bull through a thick conventional minefield as opposed to just over a third of the tanks killed by the WAMs.) This suggests that fewer red tanks arriving together in the conventional mine case were as effective at opposing the blue forces as were the more numerous, but piecemeal, red tanks in the WAM case. And since in both cases all red tanks were destroyed by the end of the battle, the only conclusion that can be drawn is that because the massed red tanks in the conventional minefield case provided more high-priority targets for the blue forces, the blue forces concentrated more of their firepower on the red tanks, foregoing and overrunning red infantry targets, thus leaving more red soldiers alive at the end of the battle. This effect may also be an artifact of running the simulation in batch mode. Human commanders would be loath to leave armed infantry dug in behind them.

Another measure of the state of the battle is the force exchange ratio (FER), which depicts the percentage of blue forces alive divided by the percentage of red forces alive. This measure can more accurately depict how the battle is progressing; whether one side or the other is gaining an advantage. Figure 12 shows the FER for the four cases. The FER at the end of the battle runs from near zero in the baseline case to near 1.0 in the conventional mine case to above 1.5 in the WAM case to above 2.0 in the no reinforcement case. The important conclusion from this chart is to note that the effect of dispersing the red tanks (as opposed to merely delaying them) is real and important in deciding the outcome of a battle. Future studies need to be performed, with interactive human decision making replacing the simple 
rules used in this study, to determine how effective WAMs are at truly dispersing a reinforcing force.

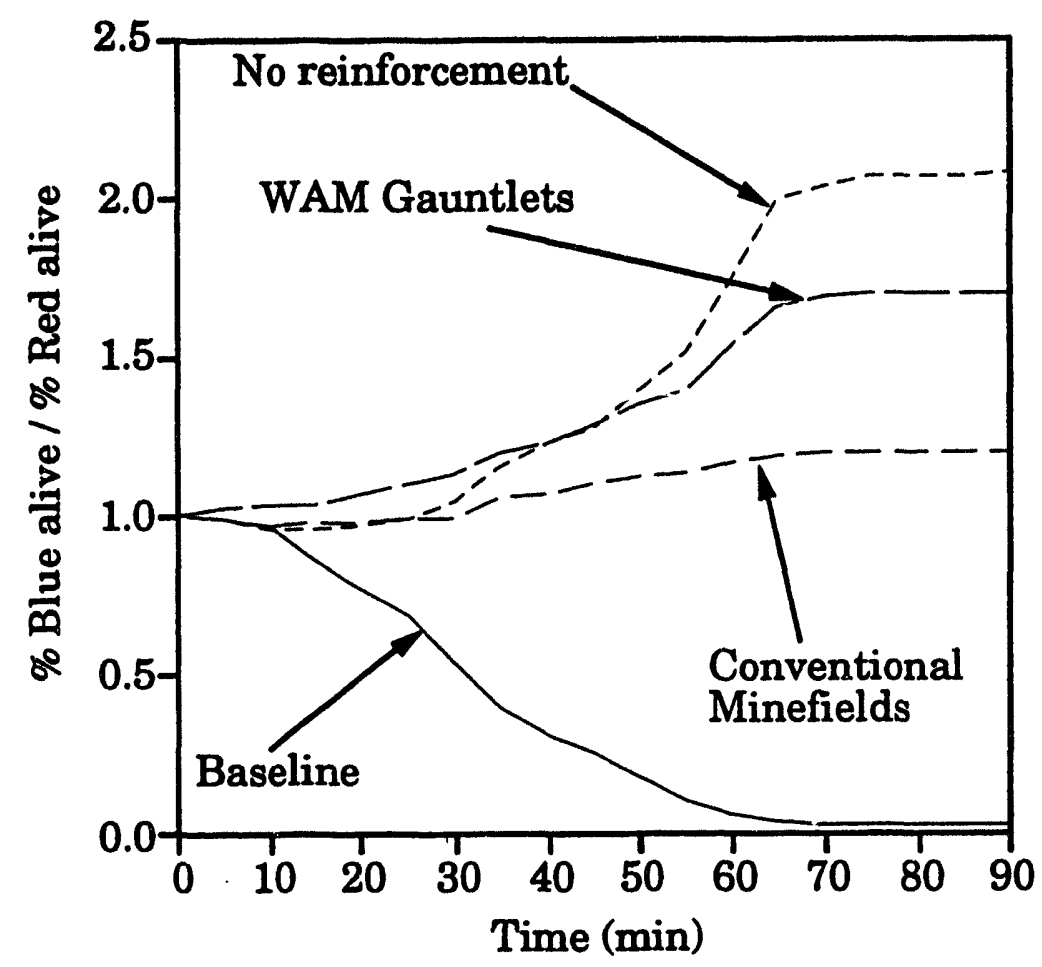

Figure 12: Mean force exchange ratios (FERs) for all four scenarios; higher numbers are better for blue.

\subsection{Conclusions}

4.1 Conclusions from the minefield model test and evaluation. The minefield model worked quite well. The entities proceeded into breach mode automatically when a minefield was detected, and then resumed normal operation once beyond the minefield. (The red tanks in the study had no breaching devices; breach mode for them entailed only different mobility characteristics ) Follow-on entities used the lane created by the lead entities. Since the dead vehicle barrier model was not included in the Janus simulation at the time these scenarios were run, dead vehicles did not clog the breach lanes. This effect was in some respects a deficiency in the simulation, and would no doubt have made the minefields more 
effective at both delaying and killing the red tanks; however, incorporating this model without a set of corresponding automated breaching tactics would have required a level of player interaction not available for this limited study.

The low-resolution WAM model worked reasonably well, providing a WAM model that was of almost equivalent resolution to the entity models. Two improvements need to be realized to make this model more acceptable. First, the WAM must be able to acquire approaching entities acoustically, not relying on lineof-sight for acquisition. Second, the effectiveness of WAM in forested and brush regions needs to be calculated and incorporated into its net effect.

The only other problem with the model, besides the lack of dead vehicles and automatic dead vehicle avoidance, was the current implementation, which was done in a prototypical fashion and which caused the code to run slowly during minefield encounters. Some minor changes to the way in which the model is implemented should suffice to ameliorate this problem.

4.2 Future activities. Both modeling and analysis activities are planned for the future. The primary modeling goal is to provide dynamic deployment capabilities for minefields, WAM, and WAM-like munitions. Deployment methods that will be included are: artillery, MLRS, helicopter, truck, and infantry. Also required is a capability for minefields to deactivate themselves either on command or after some preset time limit. Mentioned earlier were the improvements needed in the WAM model (acquisition, ground cover effects). The barrier model will be added to the Janus simulation. An algorithm to perform automatic dead vehicle avoidance has been designed, and needs to be implemented. Nevertheless, this algorithm will be very simple and will not encompass the range of possible decisions that a commander on the scene might make. For that to happen, more research in automated decision making on the tactical battlefield needs to be done. Some efforts will be expended on improving the implementation of the model, with 
performance enhancements and a more friendly user interface being the highest priorities.

On the analysis side, a more detailed analysis of the effects of delay and dispersal is required to follow up on the preliminary analysis presented here. Parameter studies of these effects using the same scenario will be needed to understand more fully what the causal relationships are between these effects, mine munitions, and the progress of a battle. Finally, the minefield and WAM models may be implemented in another simulation to allow their use in studying postulated advanced mine munitions as weapons against hardened artillery sites. 


\section{APPENDIX: MINE MODEL LOGIC}

\section{A.1 THE EFFECT OF A MINE REGION ON A PLATFORM}

Input: (region, platform type, rectangle representing entity's path)

Output: (endpoint, effect)

Place all mines in region into a mine array, sorted by distance from the vehicle

For each mine in the mine array do:

Begin Loop

Get mine type

IF mine is a individual mine ("individual mine")

Find which area of effect this mine is in

Get Pmd = prob_mine_being_destroyed(area of effect, platform type, mine type) [The probability that the platform will destroy this type of mine when the mine is encountered in a particular area of effect]

Get Pmt = prob_mine_trigger(area of effect, platform type, mine type) [The probability that this mine type will trigger when exposed to this platform.]

ELSE IF mine is a density mine

Get Pmd = probability of mine being destroyed(platform type, mine type), averaged over all areas of effect.

Get Pmt = probability of mine triggering(platform type, mine type), averaged over all areas of effect.

Get RND [a random number between 0 and 1]

IF RND $\leq$ Pmd

mine is destroyed

next mine

ELSE IF RND > Pmd

mine is not destroyed

Get new RND

IF RND > Pmt

Mine does not trigger on platform

next mine

ELSE IF RND $\leq$ Pmt

Mine triggers on platform

Mine count $=$ mine count -1

IF mine count $>0$

nothing happens

next mine

ELSE IF mine count $=0$

mine explodes

Get mpk = mine probability of kill(platform type, mine type)

Get new RND

IF $\mathrm{RND}>\mathrm{mpk}$

No kill

next mine

ELSE IF RND $\leq$ mpk

kill platform

record stopping point

End Loop exit loop

END of assessment of effect of minefield on platform 


\section{A.2 TEE EFFECT OF A PLATFORM ON A MINE REGION}

Input: (region, platform type, rectangle representing platform's path, area number of this area of effect)

Output: (changed region, number of mines left in this region)

Place into array only those mines (represented as mine density) in the region which are counting mines, i.e. have count greater than 1 , sorted by distance from vehicle. Place individual mines in region into the mine array

For each mine in the mine array do:

Begin Loop

Get mine type

Get Pmd = probability of mine being destroyed (area of effect, platform type, mine type) [Individual areas of effect this time]

Get RND

IF RND $\leq$ Pmd

mine is destroved

IF mine is individual mine

remove from minefield

next mine

ELSE IF mine is density mine ignore, since it will be taken care of in the density calculation below next mine

ELSE IF RND > Pmd

mine is not destroyed, still active

Get Pmt = probability of mine triggering(area of effect, platform type, mine type) [Individual area this time]

Get new RND

IF RND > Pmt

Mine does not trigger on platform

Leave mine alone next mine

ELSE IF RND $\leq$ Pmt

Mine triggers on platform

IF mine is individual mine

count $=$ count -1

IF count $>0$ do nothing

ELSE IF count $=0$

Remove individual mine (it exploded)

ELSE IF mine is a density mine

Get count

IF count $\leq 0$ ignore (assume explosion), it will be taken care of below next mine

ELSE IF count $>0$ create individual mine and insert it into the minefield subtract 1 /area from density

IF density $<0$ density $=0$

End Loop next mine 
For each level in region, do:

\section{Begin Loop}

Get mine type

Cet $\mathrm{Pmd}=$ probability of mine being destroyed(area, platform type, mine type)

Get Pmt = probability of mine triggering(area, platform type, mine type)

(effect of platform on mine region) $=(1-\mathrm{Pmd}) *(1-\mathrm{Pmt})$

(region's density) $=$ (region's density) * (effect of platform on mine region)

\section{End Loop}

END of assessment of effect of platform on minefield

\section{REFERENCES}

1 Dianne R. Calloway et al., The Janus Algorithms Guide, Lawrence Livermore National Laboratory Report M-227 Rev. 1, Feb. 1, 1990.

\section{ACKNOWLEDGMENTS}

The author wishes to thank Arnold Warshawsky, Stephen Wong, Jeffrey Pimper, JoAnn Matone, and Michael Uzelac for their assistance in performing this research. 

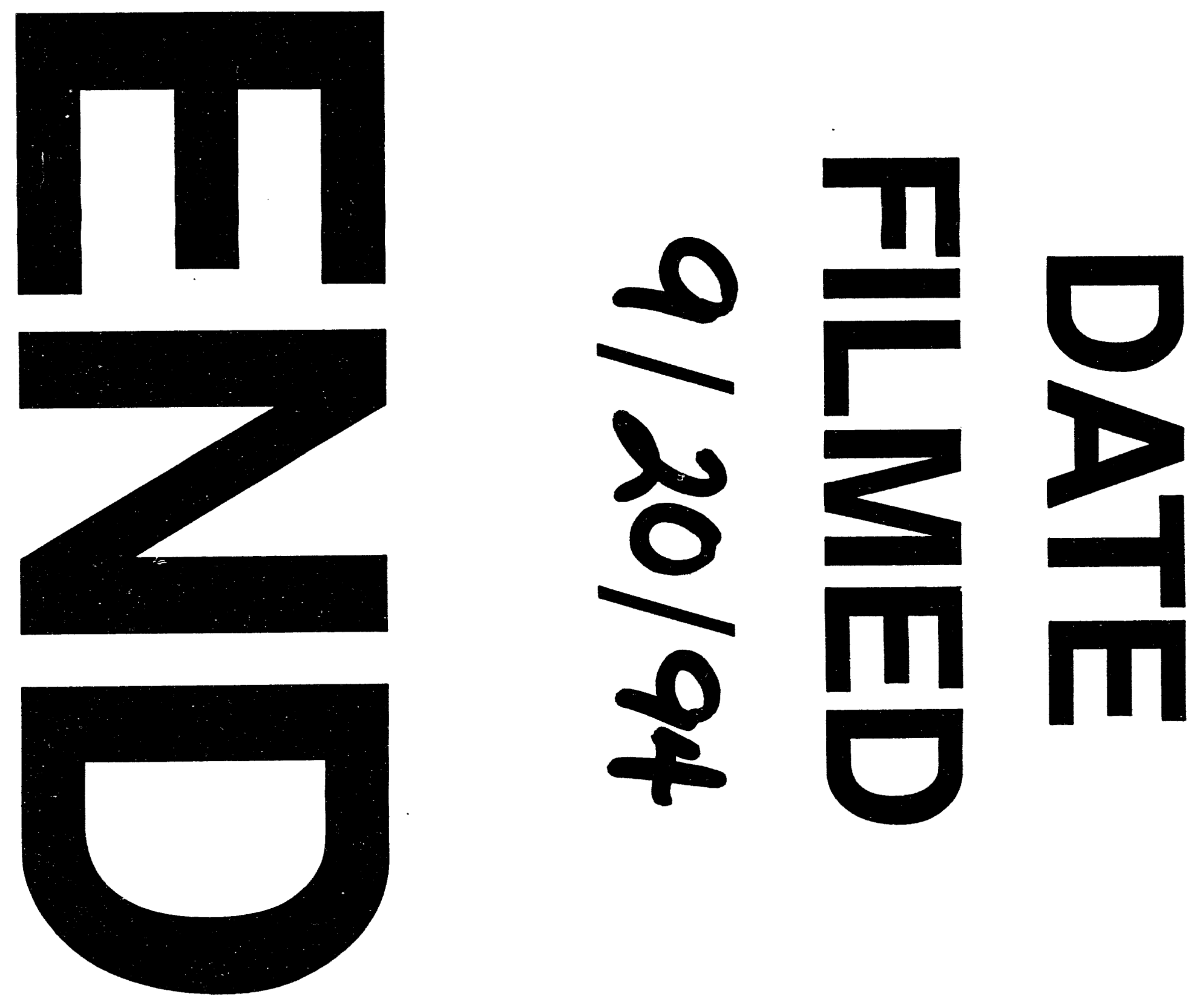